\title{
Biomarkers Expression in Cervical Cancer and High Grade Squamous Intraepithelial Lesions*
}

\author{
Ostojich Marcela ${ }^{1}$, Gimenez Liliana ${ }^{2}$, Gianni Sergio ${ }^{1}$, Alvarez Ana ${ }^{3}$, Marino Lina ${ }^{2}$, De Dios Diana ${ }^{1}$, \\ Jasnis Maria Adela ${ }^{4}$
}

${ }^{1}$ Departament of Gynecology, Institute of Oncology Angel H. Roffo, University of Buenos Aires, Argentina; ${ }^{2}$ Department of Pathology, Institute of Oncology Angel H. Roffo, University of Buenos Aires, Argentina; ${ }^{3}$ Department of Clinical Oncology, Institute of Oncology Angel H. Roffo, University of Buenos Aires, Argentina; ${ }^{4}$ Department of Immunobiology, Institute of Oncology Angel H. Roffo, University of Buenos Aires, Argentina.

Email: marcelaostojich@yahoo.com.ar

Received August $17^{\text {th }}, 2012$; revised September 19 $9^{\text {th }}, 2012$; accepted September $30^{\text {th }}, 2012$

\begin{abstract}
Objectives: The finding of new prognostic factors in human cervix cancer is necessary to improve present conventional treatments. The aim of the present study was to determine the expression and evaluate the prognostic value of hypoxia-inducible factor-1(HIF-1 $\alpha$ ), vascular endothelial growth factor (VEGF) and eritropoyetin receptor (EpoR) in cervix cancer stages IIA-IIB and in preinvasive high grade squamous intraepithelial lesions (HSIL) Methods: The study included 70 patients with cervix cancer, FIGO stages IIA-IIB, 28 patients with HSIL and normal cervix $(\mathrm{n}=28)$. HIF-1 $\alpha$, VEGF and EpoR expression were analyzed in tissue samples by immunohistochemistry using commercial antibodies. Expression and overexpression of the tumor markers were quantified according to German Immunoreactive Score. Results: HIF-1 $\alpha$, EpoR and VEGF overexpression was detected in $30 \%, 37 \%$ and $51 \%$ of cancer patients respectively. Patients with HSIL showed enhanced expression only of EpoR and VEGF (39.2\% and 71.4\%) while VEGF was overexpressed in $21 \%$ of the specimen. No correlation was found between VEGF and EpoR with disease-free overall survival (OS), tumor recurrences or prognostic factors. Only overexpression of HIF-1 was associated with less median survival measured up to 24 months, unless it was not maintained a long time. Conclusion: Although any of the markers could be considered as independent prognostic factor for cervix cancer patients, our data showed a significant increase in their expression from the premalignant lesion up to the invasive stages of tumor progression.
\end{abstract}

Keywords: Prognostic Marker; Cervical Cancer; Intraepithelial Lesion; HIF-1 $\alpha$; VEGF; EpoR

\section{Introduction}

Squamous cell carcinoma of cervix is the most frequent tumor within female genital tract and one of the leading causes of female cancer-related death. It accounts for approximately two-thirds of all cervical cancer cases, whereas adenocarcinomas and adenosquamous carcinomas account for $10 \%-25 \%$ [1]. In our country, cervical cancer shows an incidence of 17.5 per 100.000 women and 7.4 deaths per 100.000 women [2]. Invasive cervical cancer and the precursor lesions are in casual relationship with infection by oncogen human papillomavirus (HPV). In the squamous epithelium, HPV mediates cellular dysplasia and neoplasia which represent oncogenic progression [3]. Persistance of HPV infection is not sufficient to transform the epithelial host cells. Transformation by HPV depends on the oncoproteins E6 and E7, whose

*Declaration of Interest: The authors report no conflict of interest. The authors alone are responsible for the content and writing of the paper. transcription is modulated by numerous transcription factors and by epigenetic mechanisms. Thus, genetic and epigenetic alterations are involved to acquire an immortal phenotype and to further progress to an overt malignant and invasive phenotype [4,5]. The E6 and E7 proteins initiate dysregulation of cell proliferation and of apoptotic mechanisms at defined targets such as p53 and retinoblastoma $(\mathrm{Rb})$ tumor suppressor proteins, respectively. The E6 protein switches off the apoptotic protection activated by genomic instability arising as a consequence of E7 induced cell cycle activation [6]. The dramatic change in the methylation pattern of several tumors is characterized by methylation of $\mathrm{CpG}$ islands in several promoter regions. The hypomethylation (extent of incorporation of $3 \mathrm{H}$-methyl groups) increased progressively with the grade of cervical neoplasia [7].

Angiogenesis is fundamental for tumor growth since it requieres an adequate blood supply for its oxygenation and nutrition demands [6]. Early at the onset, tumor an- 
giogenesis is mediated not only by angiogenic factors but also directly by hypoxia [7-9]. As tumors grow larger, sustained tissue hypoxia may also cause molecular changes associated with a more malignant phenotype less sensitive to cytotoxic and/or radiotherapy treatments [10, 11]. Lack of oxygen is a hallmark of solid tumor formation and constitutes an independent prognostic factor in diverse malignant tumors [12] Hypoxia-inducible factor1 (HIF-1) is the transcriptional factor that mediates cellular response to hypoxia [13]. HIF-1 protein is a heterodimer composed of HIF-1 $\alpha$ and HIF-1 $\beta$ (ARNT) subunits that bind to specific hypoxia-responsive elements (HREs) and it is protected from ubiquitination and proteosomal degradation under hypoxic conditions [14, 15]. HIF1 influences the expression of a diverse set of genes associated with tumor progression, e.g. eritropoyetin (Epo), transferrin, endothelin-1, iNOS, Glut-1 transporter, VEGF and inflammatory molecules as well as pro- and antiapoptotic genes [16-18]. Cancer cervix hypoxia has been described to be independent of clinical size, grade and FIGO stage $[19,20]$ and is nowadays considered a powerful independent prognostic factor for the outcome of the disease $[11,21]$.

Vascular endothelial growth factor (VEGF) is an important mediator of tumor angiogenesis. Tumor cells within tumor microenvironment secrete VEGF under stressed conditions as hypoxia [22], radiotherapy [23] and chemotherapy [24], resulting in low response and thus poor prognosis.

Besides VEGF, erythropoietin (Epo) is another of the best known hypoxia-regulated genes mediated by HIF-1 [25]. Epo is a glycoprotein hormone that stimulates erythropoiesis, which exerts its effect by stimulating growth, preventing apoptosis and inducing differentiation of red blood cell precursors. Epo receptor (EpoR) belongs to the superfamily of cytokine receptors; Epo and EpoR signaling stimulates not only hematopoietic tissues and cells, but also a variety of solid tumors including breast and cervical cancer [26,27].

Our aim was to analyze the expression of HIF-1 $\alpha$, VEGF and EpoR in locally advanced cervical cancer and in premalignant HSIL to evaluate their value as prognostic markers of cervix neoplasias. The identification of prognostic and/or predictive biological markers in cervix cancer patients and their potential application in new and improved therapeutics would be of great importance for survival improvement.

\section{Material and Methods}

\subsection{Patients and Tissue Samples}

The retrospective study included 70 patients with cervical cancer FIGO stages IIA and IIB, treated and followed up at the Department of Gynecology, Institute of Oncol- ogy A. H. Roffo (University of Buenos Aires). Patients were randomly selected from our data base between years 1987-1999. Inclusion criteria were patients with diagnosis of uterine cervix carcinoma confirmed by an expert pathologist and further classified as stage II according to FIGO guideline; complete radiotherapy treatment at our Institute (TCT 50 Gy and BT 35 - 40 Gy); access to histological samples included in paraffin were also required. Patients of the database with no posttreatment follow up and with other oncological diseases (except basocellular tumors) were excluded. Women with high grade squamous intraepithelial lesions (HSIL) were also included in the study $(\mathrm{n}=28)$. Normal cervical tissues $(n=28)$ were obtained from hysterectomies due to benign uterine diseases. Clinico-pathological characteristics of patients are shown in Table $\mathbf{1}$. The study was approved by the Ethical Committee at the Institute of Oncology A. H. Roffo.

Table 1. Clinicopathological characteristics of patients.

\begin{tabular}{|c|c|}
\hline Median age (range) & 46 y $(30-76)$ \\
\hline Tissue Samples & $\mathbf{n}$ \\
\hline Normal cervix & 28 \\
\hline HSIL & 28 \\
\hline Cervical cancer & 70 \\
\hline \multicolumn{2}{|l|}{ FIGO stage } \\
\hline IIA & $13(18.6)$ \\
\hline IIB & $57(81.4)$ \\
\hline \multicolumn{2}{|l|}{ Histology } \\
\hline Squamous & $55(78.5)$ \\
\hline Adenoca & $14(20)$ \\
\hline $\mathrm{A} / \mathrm{s}$ & $1(1.4)$ \\
\hline \multicolumn{2}{|l|}{ Differentiation grade } \\
\hline G1 & $14(20)$ \\
\hline $\mathrm{G} 2$ & $20(28.5)$ \\
\hline G3 & $21(30)$ \\
\hline n.d & $15(21.4)$ \\
\hline \multicolumn{2}{|l|}{ Platelet count } \\
\hline$\leq 400.000$ & $33(47.2)$ \\
\hline$>400.000$ & $10(14.3)$ \\
\hline n.d & $27(38.5)$ \\
\hline
\end{tabular}

Clinicopathological charateristics of cervical cancer patients and samples size are shown. Adenoc: adenocarcinoma; A/s: adenosquamous; $\mathrm{n}$ : indicate number of patients; numbers between parenthesis indicate $\%$ of patients; n.d.: not determined. 


\subsection{Immunohistochemistry}

Formalin-fixed, paraffin-embedded 4- $\mu \mathrm{m}$ tissue sections were stained with standard immunohistochemical methods. Sections were deparaffinized and endogenous peroxidase was blocked in $3 \% \mathrm{H}_{2} \mathrm{O}_{2}$ in TBS buffer, $\mathrm{pH} 7.4$ for $10 \mathrm{~min}$. Antigen retrieval was performed using a microwave. After heating, slides were incubated with antibodies anti-HIF-1 $\alpha$ (dilution 1:50, Santa Cruz, sc-53546,) anti-VEGF (dilution 1:250, Biogenex, PV-483,) and antiEpoR (dilution 1:200, Santa Cruz, sc-695) and detected with secondary biotinilated antibodies. Diaminobenzidine was used as substrate cromogen and all slides were then counterstained with hematoxilyn. Negative controls were performed without primary antibodies.

\subsection{Immunoreactivity Score}

All sections were evaluated in a blinded manner by two pathologists. Regions of greatest immunostaining for each antibody were selected and the percentage of immunoreactive cells and staining intensity were scored according to German Immunoreactive Score. The percentage of immunoreactive tumor cells was rated as follows: no staining $=0$; up to $10 \%=1 ; 11 \%-50 \%=2 ; 51 \%$ $80 \%=3 ;>81 \%=4$; intensity: weak $(1+) ;$ moderate $(2+)$; strong $(3+)$. The grade of score expression was obtained multiplying percentage by intensity: 0 , negative; $1-4$, weak; 5 - 8, moderate, 9 - 12, strong. Overexpression was considered for scores 5 to 12 (moderate + strong).

\subsection{Statistical Analysis}

Correlation between markers (HIF-1, VEGF and EpoR) and clinicopathological variables was analyzed by Chi- square or Fisher's test. Cox regression was used to determine independent prognostic values. Overall survival analysis was performed using the Kaplan Meyer method and log-rank test was used to compare survival curves. Statistical significance was defined as $\mathrm{p}<0.05$.

\section{Results}

HIF-1 $\alpha$ was mainly identified in tumor cell cytoplasm of $39 / 70(55.7 \%)$ of cancer specimens, being overexpressed in nearly a half of them $(21 / 39,53.8 \%)$. On the other hand, barely $2 / 28$ HSIL and 1/28 normal cervix showed positive weak staining ( $\mathrm{p}<0.05$ vs cervix cancer) (Table 2, Figures 1(a)-(c)).

EpoR was not only detected in significantly higher percentage of cancer patients compared to HSIL $(72.8 \%$ vs $39.2 \%$ respectively, $\mathrm{p}<0.05)$, but half of the positive cancer samples (26/50) showed overexpression of EpoR (Figures 2(a)-(c)). Only a small number of normal cervix (17.8\%) expressed EpoR (Table 2).

A very similar high percentage of cancer and HSIL samples expressed VEGF (70\% and $71.4 \%$ respectively); however overexpression was significantly more frequent in cervix than in HSIL specimens $(73.4 \%$ vs $30 \%$ respectively, $\mathrm{p}<0.001)$. Unexpectedly, positive VEGF was detected in $35 \%$ of normal cervix ( $p<0.05$ vs HSIL). Figures 3(a)-(c) show VEGF expression in carcinoma, preinvasive and normal cervix respectively.

When we evaluated the level of expression of the combination of the three markers in cancer patients, we only found that a significant number of patients co-expressed EpoRc with HIF-1 or with VEGF $(52.8 \%$ and $57 \%$ respectively, $\mathrm{p}<0.05$ ) (Table 3).

The median survival time among the whole cohort of

Table 2. Expression of HIF-1 $\alpha$, EpoRc and VEGF.

\begin{tabular}{cccccccc}
\hline & & \multicolumn{3}{c}{ HIF1 $\alpha$} & \multicolumn{2}{c}{ EpoRc } & \multicolumn{2}{c}{ VEGF } \\
\cline { 3 - 8 } \multicolumn{2}{c}{ Tissue samples } & $(+)$ & $(++)$ & $(+)$ & $(++)$ & $(+)$ & $(++)$ \\
\cline { 3 - 8 } & & $\mathrm{n}(\%)$ & $\mathrm{n}(\%)$ & $\mathrm{n}(\%)$ & $\mathrm{n}(\%)$ & $\mathrm{n}(\%)$ & $\mathrm{n}(\%)$ \\
\hline $\mathrm{N}$ & $(\mathrm{n}=28)$ & $1(3.5)$ & - & $5(17.8)$ & - & $10(35.7)$ & - \\
$\mathrm{HSIL}$ & $(\mathrm{n}=28)$ & $2(7.2)$ & - & $11(39.2)^{\circ}$ & - & $20(71.4)^{\circ}$ & $6(30)$ \\
$\mathrm{CC}$ & $(\mathrm{n}=70)$ & $39(55.7)^{*}$ & $21(53.8)$ & $51(72.8)^{*}$ & $26(50.9)$ & $49(70)$ & $\mathbf{3 6 ( 7 3 . 4 ) ^ { \circ }}$ \\
\hline
\end{tabular}

Number (n) of patients with marker expression $(+)$ and overexpression $(++)$; numbers between parenthesis indicate $\%$ of patients; percentage of overexpression was calculated in positive samples. N: normal cervix; CC: cervical cancer; Significance between groups compared with Fisher test $(\mathrm{p}<0.05)$. HIF-1: "CC vs HSIL ( $<<0.05)$; EPORc: "HSIL vs N $(\mathrm{p}<0.05) ;$ “ $\mathrm{CC}$ vs HSIL $(\mathrm{p}<0.05) ;{ }^{\circ}$ VEGF: HSIL vs N; CC overexpression vs HSIL $(\mathrm{p}<0.05)$.

Table 3. Coexpression of EpoRc with HIF-1 $\alpha$ and with VEGF in cancer patients.

\begin{tabular}{ccccc}
\hline \multirow{2}{*}{ Biomarkers } & HIF $1 \alpha(-)$ & HIF $1 \alpha(+)$ & VEGF $(-)$ & VEGF $(+)$ \\
\cline { 2 - 5 } & $\mathrm{n}(\%)$ & $\mathrm{n}(\%)$ & $\mathrm{n}(\%)$ & $\mathrm{n}(\%)$ \\
\hline EpoRc $(+)$ & $14 / 70(20)$ & $37 / 70(\mathbf{5 2 . 8})^{*}$ & $10 / 7(14.2)$ & $40 / 70(\mathbf{5 7})^{*}$ \\
Epo Rc (-) & $17 / 70(24.3)$ & $2 / 70(2.8)$ & $11 / 70(15.7)$ & $9 / 70(13)$ \\
\hline
\end{tabular}

Table 3 shows cancer patients with co-expression of EpoR with HIF-1 and VEGF. n (\%) = number of patients (percentage). Significance with Crosstabs, Fisher's test $\left.{ }^{*} \mathrm{p}<0.001\right)$. 


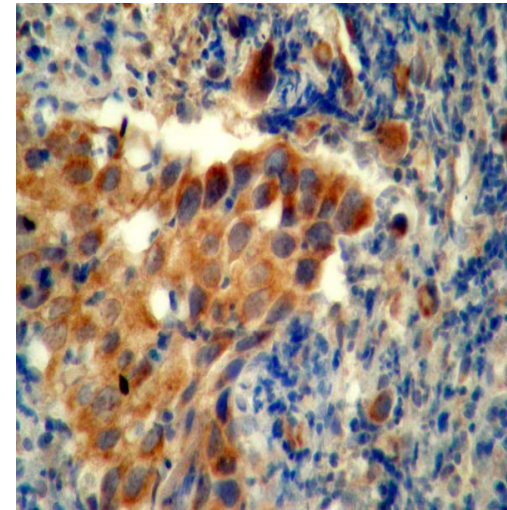

(a)

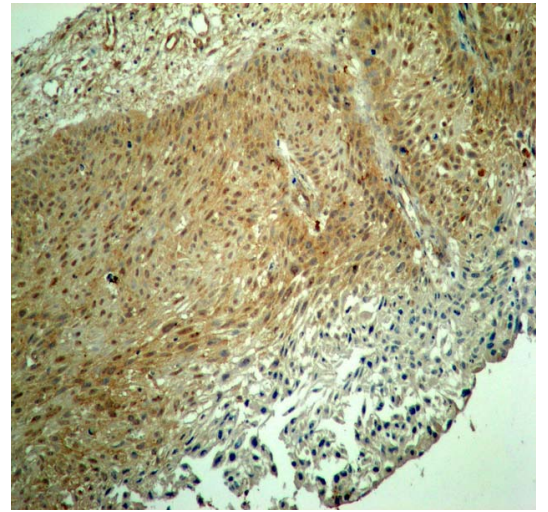

(b)

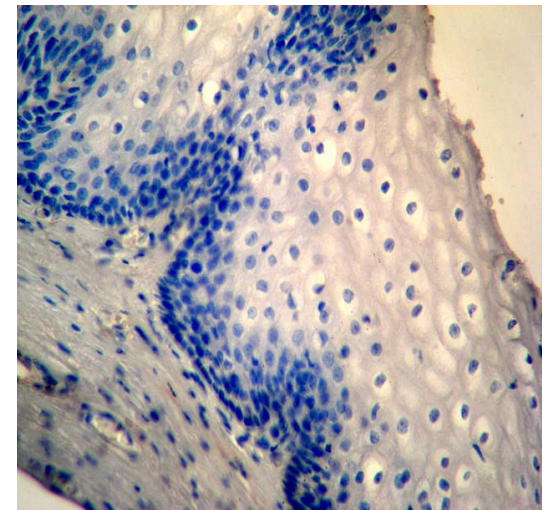

(c)

Figure 1. Immune staining for HIF-1 $\alpha$ in Cancer, HSIL and normal cervix. Immunohistochemistry of cancer, HSIL and normal tissues using specific monoclonal antibody anti HIF-1 $\alpha$. (a) Representative invasive cervix cancer showing positive strong cytoplasmatic HIF-1 $\alpha$ immunoreactivity. Some positive nucleus are also seen (400×); (b) HIF-1 $\alpha$ nuclear expression in HSIL (100×); (c) negative normal cervix (200×).

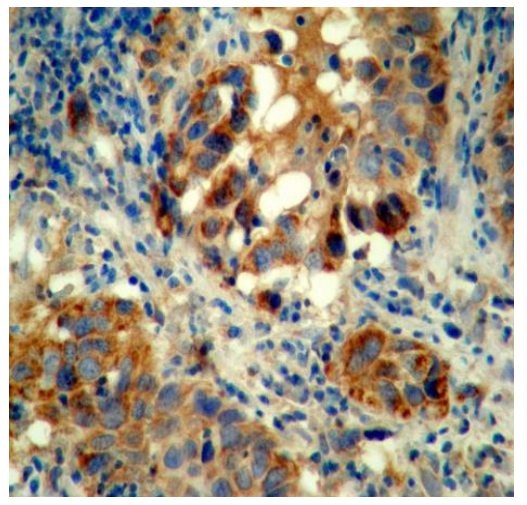

(a)

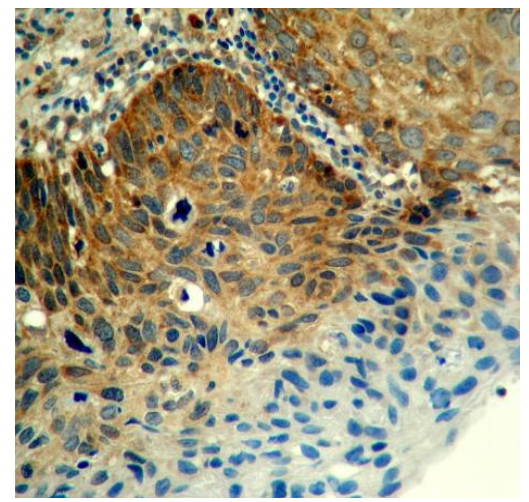

(b)

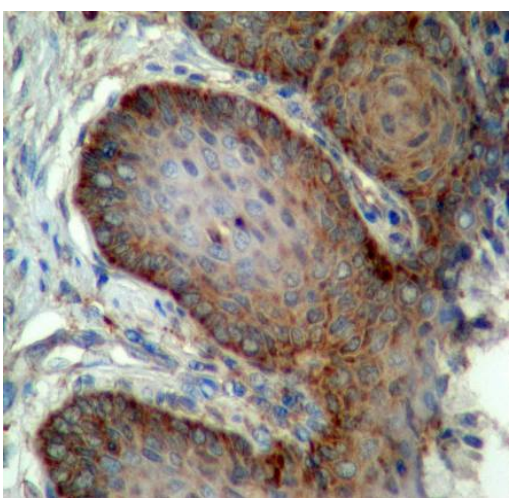

(c)

Figure 2. Immune staining for EpoRc in cancer, HSIL and normal cervix. Immunohistochemistry of cancer, HSIL and normal tissues using specific monoclonal antibody anti EpoRc. (a) Invasive cervix cancer shows intense immunostaining for EpoR (500×); (b) Representative cytoplasmatic immmunoreactivity in HSIL (400×); (c) Positive immunoreaction in normal squamous epithelial cells $(\mathbf{1 0 0} \times)$.

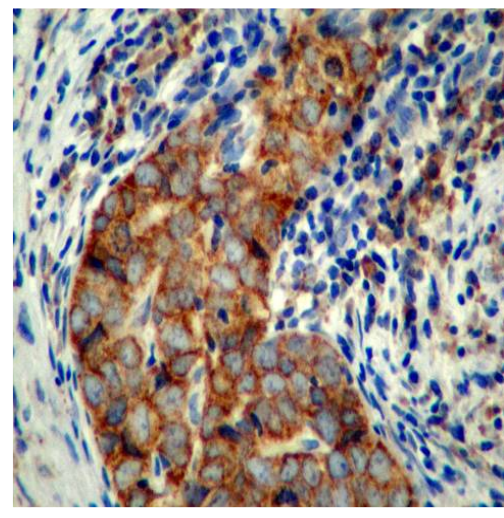

(a)

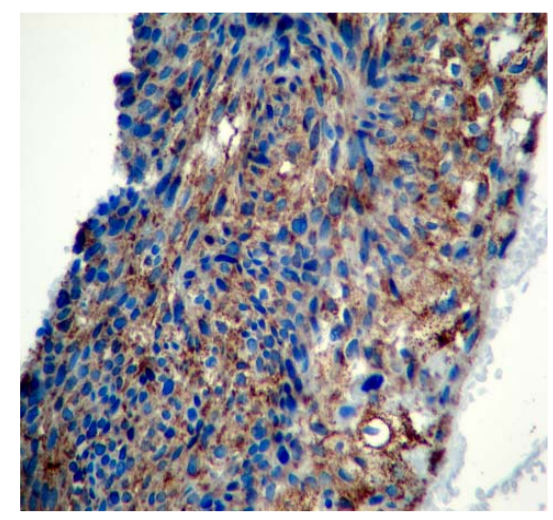

(b)

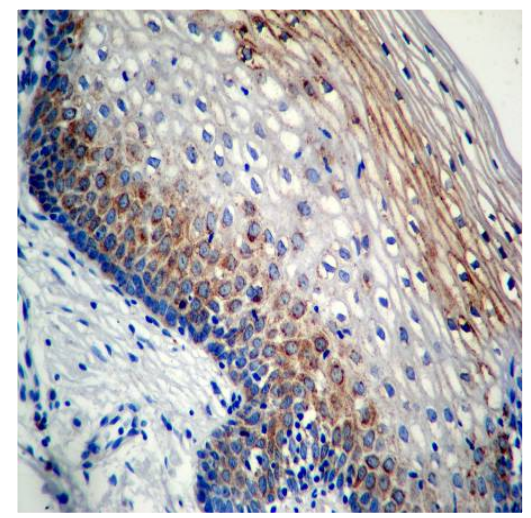

(c)

Figure 3. Inmmune staining for VEGF in cancer, HSIL and normal cervix. Immunohistochemistry of cancer, HSIL and normal tissues using specific monoclonal antibody anti VEGF. (a) Intense VEGF cytoplasmatic immunoreactivity in tumor cells from invasive cervix cancer (400×); (b) VEGF in HSIL (200×); (c) Positive immunoreactivity in normal epithelial cells $(200 \times)$. 
cancer patients was 47 months (range 8 - 195 months). We did not find any significative correlation between markers expression neither with known established prognostic factors nor with disease-free survival (DFS). Only overexpression of HIF-1 was significantly associated with median survival measured at 24 months of evolution (Kaplan-Meier, $\mathrm{p}<0.05$ ); however, at long-term, this difference was not sustained (Figures 4(a)-(c)). As expected, no recurrences were detected in patients with HSIL.

\section{Discussion}

HIF-1, VEGF and EpoRc expression in cervix cancer was compared not only with normal cervix but mainly with premalignant HSIL lesions. Our data showed positive HIF-1 nuclear and cytoplasmatic immunoreactivity of tumor cells in 39/70 cancer patients, being overexpressed in more than a half (53.8\%) (Figure 1). HSIL and normal cervix barely showed weak positive staining $(\mathrm{p}<0.05)$. Our results are in accordance with recent data reporting increased HIF- $1 \alpha$ and VHL proteins in advanced uterine cervical carcinomas compared to the corresponding normal samples and cervical intraepithelial neoplasia $[16,28]$. Overexpression of HIF- $1 \alpha$ was described in many other solid tumors such as colon, breast, skin, lung, gastric, pancreatic, prostate and renal carcinoma [11,14,29-31].

Tumor aggressiveness is associated with enhanced expression of hypoxic associated markers such as HIF-1, VEGF, GLUT-1, nitric oxide [32]. Since markers of microvessel density increased from normal epithelium to squamous cell carcinoma [33], it has been suggested that HIF- $1 \alpha$ should not be used as an endogenous marker of tumor hypoxia in locally advanced uterine squamous cell carcinomas with significant prognostic impact [34]. It is noteworthy that in our cohort of normal cervix tissue, although $35 \%$ were positive for VEGF, HIF-expression was not detected.

Analysis of overall survival (OS) followed up to 24 months (mo), showed that only HIF overexpression was significantly associated with lower median survival (12 mo vs $22 \mathrm{mo}$ ) in patients with persistent disease (Kaplan Meier $\mathrm{p}<0.05$ ). However, in long term, this difference was not sustained. Thus, HIF-1 failed to be a prognostic factor in our cohort of cervix cancer patients (Figure 4(a)).

Angiogenesis plays a key role in tumour growth and metastasis and angiogenic factors may help to identify patients with a poor prognosis [34]. Aberrant new blood vessels lead to tumour hypoxia limiting cell growth. However, tumor cells can adapt to hypoxic conditions, switching a more malignant phenotype and/or poor response to radio and chemotherapy [35]. VEGF is one of the main factors involved in tumor vascularization and

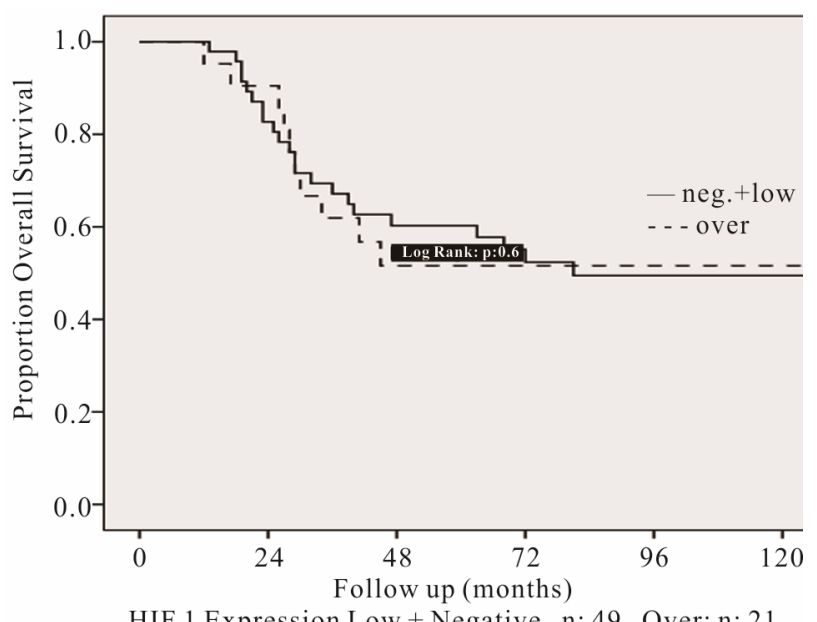

HIF 1 Expression Low + Negative n: 49 Over: n: 21

(a)

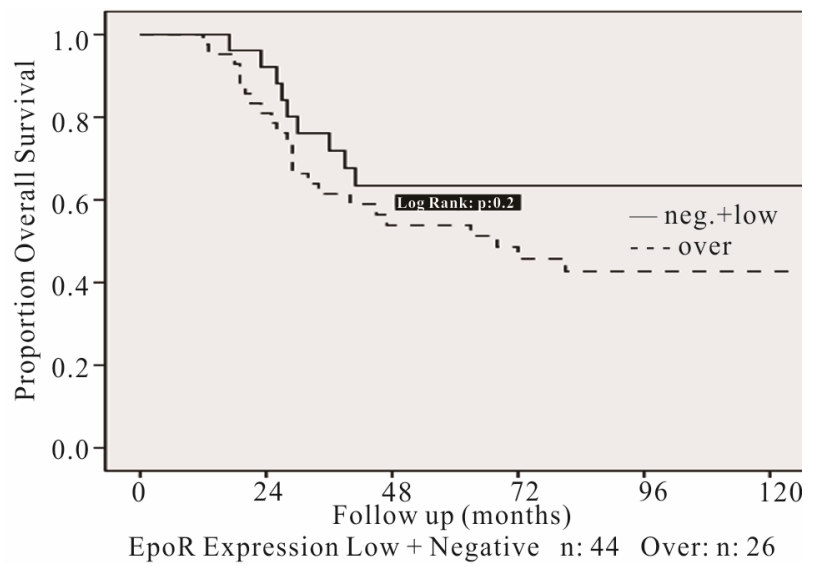

(b)

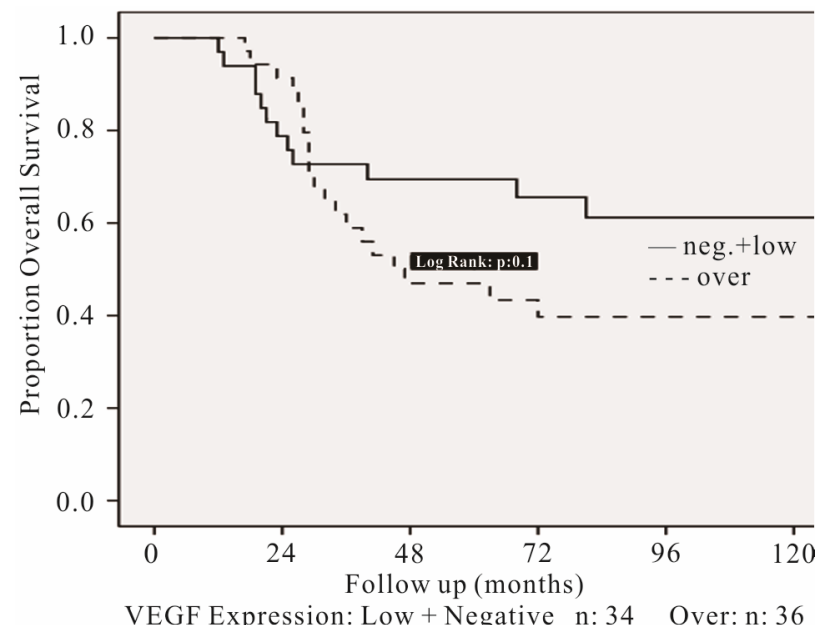

(c)

Figure 4. Overall Survival (OS) of Patients with Cervix Cancer according Biomarkers Expression. Figure 4 shows overall survival (OS) curves of cervix cancer patients followed during 120 months and according to the level of expression of HIF-1 $\alpha$ (a), EpoR (b) and VEGF (c). Patientes with overexpression (- - - over); patients with low or negative expression (- neg + low). 
can be upregulated in response to hypoxia, inducing the angiogenic switch [36]. We find almost equal expression of VEGF in cancer and HSIL samples $(70 \%$ and $71.4 \%$ respectively); however, higher number of cancer patients showed VEGF overespression (73.4\% and 30\% respectively, $\mathrm{p}<0.05$ ) (Table 2). According to our results we can assume that although VEGF is expressed in both cervix cancer and preneoplastic HSIL lesions, advanced stages of the disease are associated with overexpression of VEGF. Probably this overexpression might be linked with a more angiogenic tumor. It has been recently reported that higher endostatin and VEGF expression are indicative of advanced cervical cancer disease, and that VEGF allowed for a distinction between patients with non-invasive and those with recurrent disease [37]. In patients with cervical carcinoma, the expression of VEGF-C was also related to lymph node metastasis representing a prognostic indicator of cervical cancer [38]. Moreover, among different biological markers of angiogenesis, only VEGFR2 expression was reported as predictor of response to radio-chemotherapy in cervical cancer $[39,40]$. It has been recently reported that EGFRc is an independent prognostic factor in cervix cancer FIGO stages IIb-IV [41]. Although we have not looked for EGFRc expression, is has been described a relation between EGF system and VEGF.

Recently, expression and function of Epo and EpoR in a variety of human cancers have been reported, including solid tumors and tumor cell lines [28]. Epo and EpoR are downstream targets of HIF and can promote acquisition of malignant phenotype and exert antiapoptotic effects on certain tumor cell lines [41]. As such, treatment with rhEpo could have harmful consequences. Thus, it is of great value to determine the presence of EpoR in cancer tissues of patients using rhEpo for radio and chemotherapy-associated anemia

In our study, EpoR expression not only occurs more often in cancer patients than in HSILs $(72.8 \%$ vs $39.2 \%$, p $<0.05$ ), but is overexpressed in $50.9 \%$ of cancer samples. Less than 20\% normal epithelia showed weak staining ( $p$ $<0.05$ vs HSIL). According to these results, we can conclude that enhanced EpoR expression seemed to be associated with tumor malignancy, since it progressively augmented from normal cervix towards premalignant lesions with a further significant increase in malignant cervix cancer.

Contradictory results have been reported about the role of Epo/EpoR in tumor biology. While the involvement of Epo in angiogenesis of human gliomas has been reported [42], other authors have not detected elevated levels of EpoR in other human tumor cells [43]. Patients with uterine cervix tumors expressing high levels of Epo, had reduced overall survival, with no correlation with intratumoral hypoxia [27].
When we analyzed co-expression between the combination of the different biomarkers, a significant association was detected only between the combination of Epo expression with HIF and with VEGF, demonstrating that in cervix cancer there is a tendency to the coexpression of Epo with any of the other two biomarkers. However, decrease in median survival (at $24 \mathrm{mo}$ ) associated with markers expresison was only detected in patients overexpressing HIF-1.

In conclusion, the present study suggests that, although HIF-1 is weakly expressed in preinvasive lesions, VEGF and EpoR are already strongly expressed (are they HIFindependent?) with further enhanced expression in IIAIIB cancer samples. Thus, the three biomarkers can be associated with cervix tumor progression along the various stages of the disease. In cervical screening, it would be useful to determine biomarkers turning up already in the premalignant stage, while in invasive stages it could be necessary biomarkers of prognostic value or for specific response to therapy. Studies with larger number of patients would be necessary to determine if these molecular targets enable to be taken into account as markers of cervix tumor prognosis.

\section{Acknowledgements}

This study was supported by grant CM13-2011 from Universidad de Buenos Aires, Argentina.

\section{REFERENCES}

[1] S. H. Liyanage, C. A. Roberts and A. G. Rockall, "MRI and PET Scans for Primary Staging and Detection of Cervical Cancer Recurrence," Women's Health, Vol. 6, No. 2, 2010, pp. 251-267. doi:10.2217/whe.10.7

[2] D. De Dios, S. Gianni, M. Ostojich and C. Kremer, "Pautas en Oncología. Diagnóstico, Tratamiento y Seguimiento del Cáncer," Instituto de Oncología A H Roffo, Universidad de Buenos Aires, Buenos Aires, 2010, pp. 137-143.

[3] J. Cuzick, M. Arbyn, R. Sankaranarayanan, V. Tsu, G. Ronco, M. H. Mayrand, J. Dillner and C. J. Meijer, "Overview of Human Papillomavirus-Based and Other Novel Options for Cervical Cancer Screening in Developed and Developing Countries," Vaccine, Vol. 26, Suppl. 10, 2008, pp. K29-K41.

[4] F. Kisseljov, O. Sakharova and T. Kondratjeva, "Cellular and Molecular Biological Aspects of Cervical Intraepithelial Neoplasia," International Review of Cell and Molecular Biology, Vol. 271, 2008, pp. 35-95. doi:10.1016/S1937-6448(08)01202-1

[5] H. Von Keyserling, T. Bergmann, M. Schuetz, U. Schiller, J. Stanke, C. Hoffmann, A. Schneider, H. Lehrach, A. Dahl and A. M. Kaufmann, "Analysis of 4 Single-Nucleotide Polymorphisms in Relation to Cervical Dysplasia and Cancer Development Using a High-Throughput Ligation-Detection Reaction Procedure,” International Jour- 
nal of Gynecological Cancer, Vol. 21, No. 9, 2011, pp. 1664-1671. doi:10.1097/IGC.0b013e31822b6299

[6] A. Szalmás and J. Kónya, "Epigenetic Alterations in Cervical Carcinogenesis," Seminars in Cancer Biology, Vol. 19, No. 3, 2009, pp. 144-152. doi:10.1016/j.semcancer.2009.02.011

[7] P. M. Das and R. Singal, "DNA Methylation and Cancer," Journal of Clinical Oncology, Vol. 22, No. 22, 2004, pp. 4632-4642. doi:10.1200/JCO.2004.07.151

[8] K. L. Bennewith and S. Dedhar, "Targeting Hypoxic Tumour Cells to Overcome Metastasis," BMC Cancer, Vol. 11,2011 , pp. 504-509. doi:10.1186/1471-2407-11-504

[9] Z. K. Otrock, H. A. Hatoum, A. H. Awada, R. S. Ishak and A. I. Shamseddine, "Hypoxia-Inducible Factor in Cancer Angiogenesis: Structure, Regulation and Clinical Perspectives," Critical Reviews Oncology/Hematology, Vol. 70, No. 2, 2009, pp. 93-102. doi:10.1016/j.critrevonc.2009.01.001

[10] C. S. F. Wong, J. Sceneay, C. M. House, H. M. Halse, M. C. P. Liu, J. George, T. C. Potdevin Hunnam, B. S. Parker, I. Haviv, Z. Ronai, C. Cullinane, D. Bowtell and A. Möller, "Vascular Normalization by Loss of Siah2 Results in Increased Chemotherapeutic Efficacy," Cancer Research, Vol. 72, No. 7, 2012, pp. 1694-1705. doi:10.1158/0008-5472.CAN-11-3310

[11] P. Vaupel, "Tumor Microenvironmental Physiology and Its Implication for Radiation Oncology," Seminars in Radiation Oncology, Vol. 14, No. 3, 2004, pp. 198-206. doi:10.1016/j.semradonc.2004.04.008

[12] B. Bachtiary, M. Schindl, R. Pötter, B. Dreier, T. H. Knocke, J. A. Hainfellner, R. Horvat and P. Birner, "Overexpression of Hypoxia-Inducible Factor- $1 \alpha$ Indicates Diminished Response to Radiotherapy and Unfavorable Prognosis in Patients Receiving Radical Radiotherapy for Cervical Cancer," Clinical Cancer Research, Vol. 9, No. 6, 2003, pp. 2234-2240.

[13] G. J. Hutchison, H. R Valentine, J. A. Loncaster, S. E. Davidson, R. D. Hunter, S. A. Roberts, A. L. Harris, I. J. Stratford, P. M. Price and C. M. West, "Hypoxia-Inducible Factor 1 Alpha Expression as an Intrinsic Marker of Hypoxia: Correlation with Tumor Oxygen, Pimonidazole Measurements, and Outcome in Locally Advanced Carcinoma of the Cervix," Clinical Cancer Research, Vol. 10, 2004, pp. 8405-8412. doi:10.1158/1078-0432.CCR-03-0135

[14] P. Vaupel, "Metabolic Microenvironment of Tumor Cells: A Key Factor in Malignant Progression," Experimental Oncology, Vol. 32, No. 3, 2010, pp. 125-127.

[15] A. Unruh, A. Ressl, H. C. Mohamed, R. S. Johnson, R. Nadrowitz, E. Richter, D. M. Katschinski and R. H. Wenger, "The Hypoxia-Inducible Factor-1 Alpha Is a Negative Factor for Tumor Therapy," Oncogene, Vol. 22, No. 21, 2003, pp. 3213-3220. doi:10.1038/sj.onc.1206385

[16] G. L. Semenza, "Hypoxia-Inducible Factors: Mediators of Cancer Progression and Targets for Cancer Therapy," Trends in Pharmacol Sciences, Vol. 33, No. 4, 2012, pp. 207-214. doi:10.1016/j.tips.2012.01.005
[17] P. Vaupel, "Hypoxia and Aggressive Tumor Phenotype: Implications for Therapy and Prognosis," The Oncologis, Vol. 13, Suppl. 3, 2008, pp. 21-26. doi:10.1634/theoncologist.13-S3-21

[18] M. W. Luczak., A. Roszak, P. Pawlik, H. Kedzia, M. Lianeri and P. P. Jagodzinski, "Increased Expression of HIF- $1 \alpha$ and Its Implication in the Hypoxia Pathway in Parimary Advanced Uterine Cervical Carcinoma," Oncology Reports, Vol. 26, No. 5, 2011, pp. 1259-1264.

[19] M. Guppy. "The Hypoxic Core: A Possible Answer to the Cancer Paradox," Biochemical and Biophysical Research Communications, Vol. 299, No. 4, 2002, pp. 676-680. doi:10.1016/S0006-291X(02)02710-9

[20] W. Y. Lee, S. C. Huang, K. F. Hsu, C. C. Tzeng and W. Shen, "Roles for Hypoxia-Regulated Genes during Cervical Carcinogenesis: Somatic Evolution during the Hypoxia-Glycolysis-Acidosis Sequence," Gynecologic Oncology, Vol. 108, No. 2, 2008, pp. 377-384. doi:10.1016/j.ygyno.2007.10.034

[21] M. Höckel and P. Vaupel, "Oxygenation of Cervix Cancers: Impact of Clinical and Pathological Parameters," Advances in Experimental Medicine and Biology, Vol. 510, 2003, pp. 31-35. doi:10.1007/978-1-4615-0205-0_6

[22] C. Leo, L. C. Horn, J. Einenkel, B. Hentschel and M. Höckel, "Tumor Hypoxia and Expression of c-Met in Cervical Cancer," Gynecologic Oncology, Vol. 104, No. 1, 2007, pp. 181-185. doi:10.1016/j.ygyno.2006.07.040

[23] M. G. Noordhuis, J. J. Eijsink, F. Roossink , P. de Graeff, E. Pras, E. Schuuring, G. B. Wisman, G. H. de Bock, A. G. van der Zee, "Prognostic Cell Biological Markers in Cervical Cancer Patientes Primarily Treated with (Chemo)radiation: A Systemic Review," International Journal of Radiation Oncology, Vol. 79, No. 2, 2011, pp. 325334. doi:10.1016/j.ijrobp.2010.09.043

[24] D. H. Gorski, M. A. Beckett, N. T. Jaskowiak, D. P. Calvin, H. J. Mauceri, R. M. Salloum, S. Seetharam, A. Koons, D. M. Hari, D. W. Kufe and R. R. Weichselbaum, "Blockage of the Vascular Endothelial Growth Factor Stress Response Increases the Antitumor Effect of Ionizing Radiation," Cancer Research, Vol. 59, No. 14, 1999, pp. 3374-3378.

[25] P. Gallet, B. Phulpin, J. L. Merlin, A. Leroux, P. Bravetti, H. Mecellem, N. Tran and G. Dolivet, "Long-Term Alterations of Cytokines and Growth Factors Expression in Irradiated Tissues and Relation with Histological Severity Scoring," PLoS One, Vol. 6, 2011, Article ID: e29399. doi:10.1371/journal.pone.0029399

[26] P. Albertsson, B. Lennernäs and K. Norrby, "Low-Dosage Metronomic Chemotherapy and Angiogenesis: Topoisomerase Inhibitors Irinotecan and Mitoxantrone Stimulate VEGF-A-Mediated Angiogenesis," Acta Pathologica, Microbiologica et Immunologica Scandinavica, Vol. 120, No. 2, 2012, pp. 147-156. doi:10.1111/j.1600-0463.2011.02830.x

[27] C. Leo, L. C., Horn, C. Rauscher, B. Hentschel, A. Liebermann, G. Hildebrandt and M. Höckel, "Expresion of Erythropoietin and Erytropoietin Receptor in Cervical Cancer and Relationship to Survival, Hypoxia and Apoptosis," Clinical Cancer Research, Vol. 12 , No. 23, 2006, 
pp. 6894-6900. doi:10.1158/1078-0432.CCR-06-1285

[28] G. Acs, P. Acs, S. M. Beckwith, R. L. Pitts, E. Clements, K. Wong and A. Verma, "Erythropoietin and Erythropoietin Receptor Expression in Human Cancer," Cancer Research, Vol. 61, 2001, pp. 3561-3565.

[29] H. H. Jo, S. H. Kim, I. A. Park, D. Kang, S. S. Han, J. W. Kim, N. H. Park, S. B. Kang and Y. S. Song, "Expression of Vascular Endothelial Growth Factor and Hypoxia Inducible Factor- $1 \alpha$ in Cervical Neoplasia," Annals of the NY Academy of Science, Vol. 1171, 2009, pp. 105-110. doi:10.1111/j.1749-6632.2009.04891.x

[30] K. Ruan, G. Song and G. Ouyang, "Role of Hypoxia in the Hallmarks of Human Cancer," Journal of Cellular Biochemistry, Vol. 107, No. 6, 2009, pp. 1053-1062. doi:10.1002/jcb.22214

[31] J.-W. Kim, P. Gao and C. V. Dang, "Effects of Hypoxia on Tumor Metabolism," Cancer Metastasis Review, Vol. 26, No. 2, 2007, pp. 291-298. doi:10.1007/s10555-007-9060-4

[32] J. Mazibrada, M. Rittà, M. Mondini, M. De Andrea, B. Azzimonti, C. Bogogna, M. Ciotti, A. Orlando, N. Surico, L. Chiusa, S. Landolfo and M. Gariglio, "Interaction between Inflammation and Angiogenesis during Different Stages of Cervical Carcinogenesis," Gynecologic Oncology, Vol. 108, No. 1, 2008, pp. 112-120. doi:10.1016/j.ygyno.2007.08.095

[33] A. Mayer, A. Wree, M. Höckel, C. Leo, H. Pilch and P. Vaupel, "Lack of Correlation between Expression of HIF-1alpha Protein and Oxygenation Status in Identical Tissue Areas of Squamous Cell Carcinomas of the Uterine Cervix," Cancer Research, Vol. 64, No. 16, 2004, pp. 5876-5881. doi:10.1158/0008-5472.CAN-03-3566

[34] S. Chouaib, Y. Messai, S. Couve, B. Escudier, M. Hasmin and M. Z. Noman, "Hypoxia Promotes Tumor Growth in Linking Angiogenesis and Immune Escape," Frontiers in Immunology, Vol. 3, No. 21, 2012, pp. 1-10.

[35] E. B. Rankin and A. J. Gaiaccia, "The Role of Hypoxia-Inducible Factors in Tumorigenesis," Cell Death and Differentiation, Vol. 15, No. 4, 2008, pp. 678-685. doi:10.1038/cdd.2008.21

[36] D. Liao and R. S. Johnson, "Hypoxia: A Key Regulator of Angiogenesis in Cancer," Cancer Metastasis Review, Vol. 26, No. 2, 2007, pp. 281-290. doi:10.1007/s10555-007-9066-y

[37] S. Landt, H. Heidecke, C. Reuter, S. Korlach, J. U. Blohmer, W. Lichtenegger, T. Heusner, F. Stoblen, M. Thill, J. Barinoff, J. Sehouli and S. Kümmel, "The Utility of an in Vitro Angiogenesis Score for Prognosis Assessment in Patients with Cervical Cancer," Anticancer Research, Vol. 31, No. 8, 2011, pp. 2645-2649.

[38] D. M. Ma, Y. P. Xu and L. Zhu, "Expression of Vascular Endothelial Growth Factor C Correlates with a Poor Prognosis Based on Analysis of Prognostic Factors in Patients with Cervical Carcinomas," Journal of Obstetrics Gynaecology Research, Vol. 37, No. 11, 2011, pp. 15191524. doi:10.1111/j.1447-0756.2011.01566.x

[39] V. M. Nagy, R. Buiga, I. Brie, N. Todor, O. Tudoran, C. Ordeanu, P. Virág, O. Tarta, M. Rus and O. Balacescu, "Expression of VEGF, VEGFR, EGFR, COX-2 and MVD in Cervical Carcinoma, in Relation with the Response to Radio-Chemotherapy," Romanian Journal of Morphology and Embryology, Vol. 52, No. 1, 2011, pp. 53-59.

[40] P. Adimi, K. D. Steffensen, D. Schledermann, E. R. Rasmussen and A. Jakobsen, "The Prognostic Importance of EGFR and COX-2 Expression in Cervix Cancer Stages IIb-IVa," Journal of Cancer Therapy, Vol. 2, No. 1, 2011, pp 9-15. doi:10.4236/jet.2011.21002

[41] E. Abhold, E. Rahimy, J. Wang-Rodriguez, K. J. Blair, M. A. Yu, K. T. Brumund, R. A. Weisman and W. M. Ongkeko, "Recombinant Human Erythropoietin Promotes the Acquisition of a Malignant Phenotype in Head and Neck Squamous Cell Carcinoma Cell Lines in Vitro," BMC Research Notes, Vol. 4, 2011, p. 553. doi:10.1186/1756-0500-4-553

[42] B. Nico, T. Annese, D. Guidolin, N. Finato, E. Crivellato and D. Ribatti, "Epo Is Involved in Angiogenesis in $\mathrm{Hu}-$ man Glioma," Journal of Neurooncology, Vol. 102, No. 1, 2011, pp. 51-58. doi:10.1007/s11060-010-0294-6

[43] A. Sinclair, N. Rogers, L. Busse, I. Archibeque, W. Brown, P. Kassner, J. E. V. Watson, G. E. Arnold, K. C. Q. Nguyen, S. Powers and S. Elliott, "Erythropoietin Receptor Transcription Is neither Elevated nor Predictive of Surface Expression in Human Tumour Cells," British Journal of Cancer, Vol. 98, No. 6, 2008, pp. 1059-1067. doi:10.1038/sj.bjc. 6604220 